\title{
Enquête
}

Archives de la revue Enquête

5 | 1989

Biographie et cycle de vie

\section{Rapports de générations et parcours de vie}

\section{Claudine Attias-Donfut}

\section{(2) OpenEdition}

\section{Journals}

Édition électronique

URL : http://journals.openedition.org/enquete/82

DOI : 10.4000/enquete.82

ISSN : 1953-809X

Éditeur :

Cercom, Éditions Parenthèses

Édition imprimée

Date de publication : 2 mars 1989

\section{Référence électronique}

Claudine Attias-Donfut, « Rapports de générations et parcours de vie », Enquête [En ligne], 5| 1989, mis en ligne le 27 juin 2013, consulté le 10 décembre 2020. URL : http://journals.openedition.org/enquete/ 82 ; DOI : https://doi.org/10.4000/enquete.82 


\title{
Rapports de générations et parcours de vie
}

\author{
Claudine Attias-Donfut
}

1 Par rapport au titre initialement prévu, je vais quelque peu modifier ma communication pour tout d'abord cerner la notion même de génération avant d'aborder les relations entre parcours de vie et rapports inter-générations.

2 Régine Robin m'a, en effet, demandé d'essayer de clarifier cette notion qui est très complexe, polysémique et qui fait l'objet de controverses importantes. Pour cela, je procéderai à un retour en arrière, en recherchant les divers usages de la notion de génération au cours de différentes périodes et en prêtant attention aux évocations significatives de certaines étapes de vie, liées à la notion de génération.

3 L'idée directrice en est la question de la formation même d'une génération. Comment se forme-t-elle dans sa dimension généralement évoquée, la dimension symbolique et, pourrait-on dire, la dimension cognitive? L'appartenance à une même génération implique des valeurs, des mentalités communes, une certaine vision du monde, c'est là l'idée directrice qui m'a conduite à entreprendre une recherche historique pour élucider les différents usages de la notion de génération.

4 De ce survol historique se dégagent deux grandes périodes : celle qui va du milieu du XIX siècle au début $\mathrm{du} \mathrm{xx}^{\mathrm{e}}$ siècle, et une seconde période, des années cinquante à nos jours. Il $\mathrm{y}$ a discontinuité entre ces deux périodes et j'essaierai d'en proposer quelques hypothèses explicatives.

5 La génération est, bien entendu, une idée universelle. On a toujours compté le temps par générations. Je ne rappellerai pas les mythologies, les conceptions philosophiques ou religieuses qui ont recours à l'idée de génération comme mesure du temps historique. Je ne traiterai de ce concept qu'à partir du moment où cette idée a été systématisée, au XIX siècle, pour comprendre les rythmes et le développement de l'histoire. Il est intéressant de reprendre ses principales théorisations, parce que la façon dont on utilise la notion de 
génération aujourd'hui est l'héritage accumulé de toutes les significations qui lui ont été données, depuis les temps immémoriaux, et en particulier, depuis le XIX ${ }^{\mathrm{e}}$ siècle.

6 Au cours de cette première période, la génération représente un instrument de mesure du temps historique, historiens et philosophes vont s'efforcer de la conceptualiser, de la systématiser, pour avoir une appréhension plus objective et plus rigoureuse de l'histoire. Cet usage de la notion de génération correspond à la naissance de l'histoire critique. Pour Auguste Comte, le progrès de l'histoire se fait dans la continuité des générations, et son rythme d'évolution est donné par le rythme de succession des générations. Lorsque ce rythme est accéléré, les générations étant plus courtes (durée de vie courte), le changement est plus rapide et lorsque les générations sont plus longues (espérance de vie plus longue), le changement est plus lent. En conséquence, plus la succession des générations est rapide, plus sensible est le progrès. Mais il nuance son propos en disant qu'une certaine continuité, donc une durée de vie relativement longue, est nécessaire pour asseoir les changements, les stabiliser, car les durées de vie trop courtes, au contraire, ne permettent pas de réels changements et donc ne favorisent pas le progrès de la société.

7 Cette idée a été diversement reprise par différents auteurs, j'en mentionnerai juste quelques éléments. Des cycles historiques ont été recherchés dans des regroupements de générations; pour Cournot, par exemple, des changements significatifs interviennent toutes les trois générations. Pourquoi trois générations? Parce qu'il y a, à un même moment, trois générations en présence qui s'influencent réciproquement, s'imprègnent mutuellement. Les jeunes sont influencés par les deux générations antérieures et c'est alors le passage de trois générations qui détermine les changements importants, ce qui correspond à environ un siècle, une génération étant évaluée à trente ans. Il faut remarquer que ces trente ans correspondent à la période de vie adulte entre 30 et 60 ans. C'est là une idée qu'on retrouve absolument chez tous les auteurs jusqu'au début du $\mathrm{xx}^{\mathrm{e}}$ siècle : le temps utile de la génération est la période de la phase de vie adulte. La jeunesse n'est pas prise en compte, quant à la vieillesse, elle est laissée de côté non seulement parce que l'espérance de vie est courte, mais aussi parce que les vieux sont relégués hors de la vie civile. La période utile est donc cette période entre 30 et 60 ans. Il y a du reste une expression très significative donnée par un des historiens de l'époque qui parle de «naissance et mort sociale » à propos de cette période de 30 à 60 ans pour la différencier de l'ensemble de l'existence de la naissance à la mort physiologique.

Dans cette conception, la génération se limite donc précisément à cette période de la vie adulte. Elle représente ce qu'on a appelé, ce matin, la cohorte, c'est-à-dire l'ensemble des personnes qui sont nées à peu près à la même époque et qui traversent ensemble une même période historique. Dilthey, par contre, considère que la génération signifie et connote un rapport de contemporanéité qui se traduit par des liens qualitatifs entre ses membres qui ont vécu ensemble des expériences, des changements sociaux. Il restreint la génération à quelques grands noms, qui se connaissent, s'influencent et marquent une époque, la signent de leur nom. Pour Dilthey donc la génération est cet ensemble de grands esprits qui ont marqué leur époque et les changements sociaux qui s'y sont opérés.

Il est du reste le seul à limiter ainsi la génération à un groupe restreint. Mais l'idée de lien qualitatif qu'il a initiée est restée et on la retrouve dans toutes les théories ultérieures, au début du $\mathrm{xx}^{\mathrm{e}}$ siècle, qui ont défini la génération par un rapport particulier au changement social et au changement historique et par l'imprégnation résultant de ces mêmes changements. 
10 Je ne m'attarderai pas sur les différents autres aspects des théories, cycles de quatre générations ou générations conçues comme généalogies avec recherches de l'hérédité des familles régnantes pour comprendre le mouvement historique et l'esprit d'une nation. Il y a eu de nombreuses recherches à cette époque-là, mais ce qui est intéressant c'est de retenir les liens entre la théorie du changement social et le concept de génération qui constituent l'héritage que Mannheim nous a légué.

11 Mannheim, en effet, a hérité de toute cette réflexion et on la retrouve dans sa théorie. Je ne pourrai, faute de temps, développer une critique de la théorie de Mannheim dont je voudrais simplement rappeler les grandes lignes. Mannheim définit une génération à partir de quatre niveaux.

12 Il y a tout d'abord ce qu'il appelle la "génération potentielle ", génération à base biologique; c'est l'ensemble des personnes nées à une même époque et qui ne forment pas nécessairement une génération d'un point de vue sociologique, sauf en période de déstabilisation sociale ou de changements sociaux, la génération potentielle s'actualisant alors en "génération effective ». La génération effective est marquée par des visions du monde contrastées, qui sont symbolisées par ce qu'il appelle des « unités de génération ». Les unités de génération ce sont, à son époque, les romantiques d'un côté, les positivistes de l'autre. Lors des événements de mai 1968, ça aurait été les fachos et les maos, par exemple. Les unités de génération discutent, traitent d'un même problème, mais en donnent des solutions différentes, des idéologisations différentes. Ces unités de génération s'expriment dans le cadre de "groupes concrets »: tels sont les quatre niveaux de sa définition. Le lien qu'il établit entre la théorie du changement social et le concept de génération se fait grâce à ce qu'il appelle le « nouvel accès à la culture », et qui émane principalement de la jeunesse.

13 Il est le premier à avoir donné à la jeunesse une importance primordiale. La jeunesse acquiert une force politique dans le processus de renouvellement des générations, en raison du contact qu'elle entretient avec le changement. Les jeunes plus réceptifs aux changements sociaux lui donnent un certain retentissement. Par le biais de ce contact neuf, de ce renouvellement perpétuel des générations, les périodes de déstabilisation trouvent là une accroche, car les jeunes s'emparent des potentiels de changement et en font un changement effectif.

14 Dans cette théorie, on voit bien que la génération est en fait déduite de l'histoire, déduite de l'observation des changements historiques. C'est la tendance qui domine toute cette période: c'est en fait de l'observation de l'histoire qu'on déduit l'existence de telle génération significative et que sont déduites les chronologies des générations. Dans la mesure où le projet théorique était précisément de comprendre les mouvements historiques grâce à la notion de génération, il y a là une sorte de raisonnement en cercle vicieux, puisque la génération qui devait expliquer l'histoire en est en fait déduite. On observe l'histoire, on dégage des moments significatifs, on en déduit l'existence d'une génération. Comment alors l'appliquer à nouveau à l'histoire pour en comprendre le mouvement?

15 On retrouve régulièrement cette difficulté quand il faut établir d'une façon concrète une méthode historique des générations, et on peut dire que durant toute cette période ce projet théorique a échoué. C'est là je pense un élément qui peut expliquer l'éclipse de cette notion de génération à partir des années 1920-1930, à l'époque de Mannheim; 
pendant une génération, la génération est devenue un concept complètement oublié, jusqu'aux années 1950.

16 Autre hypothèse, pour expliquer cette éclipse, le fait que la notion de génération s'inscrit dans le cadre d'une conception de l'histoire qui fait des idées, de la science, de la marche de l'esprit, le moteur principal de l'histoire. L'histoire est perçue comme unidimensionnelle, son rythme et son évolution sont donnés par l'évolution de l'esprit qui est concrétisée par les générations. Reconstituer une histoire par génération, c'est considérer une histoire faite d'hommes qui pensent et qui, par le mouvement de la pensée, font changer la société. Les développements de l'économie, l'importance acquise par les théories marxistes ont donné beaucoup plus d'importance aux moyens de production au détriment de la superstructure. En outre, la prise de conscience d'une temporalité multiple en histoire a également contribué à reléguer toute cette conception de l'histoire et avec elle, la notion même de génération.

17 L'utilisation de la génération a, par ailleurs, correspondu à l'illusion de trouver des lois en histoire, illusion qui a été abandonnée également au début du siècle. En histoire comme en théorie des sciences, une transformation profonde a contribué à reléguer toutes ces théories des générations qui n'ont été exhumées qu’à partir des années 1950, dans de tout autres directions.

18 J'en arrive à la seconde période durant laquelle l'histoire a complètement évacué la notion de génération, mais la sociologie l'a alors récupérée avec cet héritage et ces connotations. La génération a été utilisée plutôt dans une optique classificatoire, dans le cadre d'une étude de la structure sociale. Les générations sont représentées comme des groupes sociaux qui entrent en interaction et structurent la société. Notion d'âge et notion de génération se rapprochent. Le retour, si l'on peut dire, de la notion de génération a coïncidé avec les premières réflexions sur les mouvements de jeunes qui ont commencé dans les années 1950 et qui ont conduit à s'interroger sur l'origine de la formation de ces groupes sur la base de l'âge et sur leur signification. On en revient en quelque sorte à Mannheim, notion de jeunesse et notion de génération étant étroitement associées. C'est là la différence par rapport à la période antérieure où la notion de génération renvoyait à la vie adulte ; depuis les années 1950, la génération est synonyme de jeunesse. Actuellement, une certaine évolution se fait jour.

D'autres sources ont été utilisées, notamment les sources anthropologiques. Eisenstadt, dans son livre From Generation to Generation ${ }^{1}$, visait à comprendre les mouvements de jeunes dans les pays occidentaux et il a pris comme modèle les analyses qui ont été faites par les anthropologues sur les systèmes de classe d'âge, surtout en Afrique. La génération est prise comme classe d'âge et, paradoxalement, les dimensions temporelle et historique sont évacuées. Il s'agit d'un groupe social et c'est en tant que tel qu'il est étudié, avec des glissements continuels entre les notions d'âge et de génération qui font jusqu'à présent l'objet de débats importants.

20 Je ne m'attarderai pas sur cette période, puisqu'on a traité ce matin des théories de parcours de vie dans ses différentes dimensions, du développement individuel ou institutionnel. Je voudrais surtout insister sur ce que je pourrais appeler le modèle à trois temps, qui consiste à vouloir différencier effet d'âge, effet de génération, effet de période. Ce modèle est très souvent utilisé et il a été mis en place précisément à la suite de cette contamination des notions d'âge et de génération, et de la difficulté à les dissocier. Ce modèle présuppose qu'il existe un effet d'âge relativement autonome qu'on pourrait mesurer en égalisant les effets de génération ou de période, ainsi qu'un effet de 
génération, donné une fois pour toutes, que l'on retrouverait à différentes périodes. Il existerait, en outre, une certaine autonomie des effets d'âge et de génération par rapport à une période donnée qui influence simultanément toutes les générations à un moment donné.

Ces présupposés implicites ou explicites qui sont à la base de ce modèle à trois temps me paraissent tout à fait contestables, car dans le processus temporel âge et génération sont très intimement interpénétrés et la période est ce qui nourrit les uns et les autres. Le vieillissement, on l'a dit ce matin, n'est pas le même d'une génération à l'autre. Les parcours de vie se transforment donc d'une génération à l'autre, les effets de vieillissement même ne se retrouvent pas identiques d'une génération à l'autre. Il n'existe pas d'effet de vieillissement pur de tout effet de génération ou de tout effet historique. De même, qu'est-ce qu'un effet de génération ? Est-ce qu'une génération est achevée à un moment donné ? Est-ce qu'on peut dire que c'est à une période de la jeunesse que la génération naît et perdure et que cet effet-là va rester cristallisé pendant toute la vie ? C'est un peu ce que Mannheim dit, et on peut le contester. On pourrait, en effet, au contraire, et c'est ce que je développerai dans quelques minutes, penser que la génération se construit à mesure de son vieillissement, et que l'effet de génération est continuellement en restructuration. Ce sont précisément les effets de période qui nourrissent les uns et les autres, le vieillissement et l'appartenance à la génération.

Bien entendu, lorsque je critique ce modèle je ne veux pas dire, pour autant, qu'il ne soit pas opératoire de tenir compte de toutes ces dimensions, âge, génération, période. Mais en tenir compte dans un but opératoire ne signifie pas qu'il faille chercher à retrouver des effets purs, comme c'est le cas dans un certain nombre de travaux. Beaucoup de travaux américains, quelques travaux français aussi usant de méthodologies extrêmement sophistiquées, s'appuyant sur des enquêtes longitudinales, sur plusieurs cohortes, se donnent comme objectif d'isoler les effets de génération ou d'âge, comme s'ils étaient autonomes. De mon point de vue, il n'existe pas d'effets purs. Et ce qui est intéressant, c'est précisément, tout en prenant ces trois dimensions, d'en analyser les interactions. C'est ce qui est le plus riche et me semble aussi correspondre davantage à la réalité : depuis plus de vingt ans que ce modèle existe et qu'il fait l'objet de recherches, il n'y a pas, je crois, une seule recherche qui a réussi à isoler des effets purs. Je pense que c'est assez significatif.

À partir de cette critique il convient de déconstruire cette dissociation d'âge et de génération, de déconstruire également, je vais être très rapide puisque je crois qu'il ne me reste plus tellement de temps, de déconstruire donc les définitions sociales des générations qui, à l'instar des théories héritées du XIX siècle, ont tendance à cristalliser une génération à un moment donné. Aujourd'hui, l'actualité est à nouveau la génération de 1968 ; cette dernière est vraiment très caractéristique de ces définitions sociales de génération qui, en fait, sont un symbole social. Il y aurait un événement donné qui, à partir de sa production, définirait une génération entière dans toute son hétérogénéité sociale et dans tout son parcours de vie. N'y a-t-il pas d'autres expériences préalables qui soient entrées en interaction avec cet événement de 68 , ainsi que d'autres événements intervenus après? En outre, il y a différentes façons de vivre un événement et ceux qui avaient 20 ou 30 ans en 68 ne sont guère homogènes à cet égard. Les discours sociaux du type de ceux qui sont tenus sur la génération de 68 méritent de mon point de vue d'être considérés comme des pratiques sociales en soi. Leur analyse relève de la construction 
continue du temps social qui se fait à l'intersection de l'histoire contemporaine et de la mémoire collective.

C'est là un thème que je développe dans mon livre, mais je n'irai pas plus loin présentement. Je vais maintenant aborder le point que je voulais traiter préalablement, sur lequel devait porter mon intervention, à savoir réintroduire les rapports entre âge et génération à la fois pour comprendre les processus de formation d'une génération et pour situer aussi le parcours de vie dans le cadre des rapports intergénérationnels. Je pars de deux constats d'évidence : 1 ) chaque étape de vie exprime un certain état des rapports entre générations ;2) une génération se produit elle-même à mesure de son vieillissement et par rapport aux autres générations. Les générations se définissent toujours les unes par rapport aux autres.

25 À titre d'exemple je mentionnerai l'adolescence et ce que j'ai appelé la maturescence, deux temps forts de confrontation intergénération, les rapports entre adolescents et adultes. Ce temps de l'adolescence, où s'ouvre une nouvelle dimension de l'existence et s'opère un engagement progressif dans la vie sociale, est le signe d'une conscience nouvelle du temps qui se crée à cette étape de la vie et d'une autonomisation par rapport aux adultes. C'est donc un temps de redéfinition des rapports entre générations qui s'accompagne d'une nouvelle conscience du temps, la conscience de génération. La création, l'apparition nouvelle d'une génération procède à la fois de cette conscience de génération et de la prise de conscience du temps historique. Par référence à l'épistémologie génétique pour caractériser les différents niveaux de l'acquisition du temps, une autre forme de temporalité abstraite est acquise : l'historicité, médiatisée par la conscience de génération. L'historicité est dans une définition très générale, la conscience de la relativité de sa propre temporalité par rapport à celle de la société ; elle est une condition essentielle de l'identité sociale. La conscience de son propre temps et la situation par rapport à sa temporalité qui est nécessairement médiatisée par la conscience de génération, apparaît à l'adolescence. Je formule l'hypothèse que son apparition est favorisée par le conflit larvé ou explicite avec la génération des adultes, la génération antérieure ; je me réfère à la théorie du conflit socio-cognitif comme condition du progrès de l'intelligence: la conscience de génération se forme d'abord contre la précédente génération, c'est une conscience, une définition contre. Et par le même processus, la génération antérieure commence à élaborer son image sociale. Ce n'est pas par hasard si vingt ans après on redéfinit les contours de la génération des soixantehuitards; leur image sociale se précise précisément parce qu'une nouvelle génération arrive et que, pour se définir, elle a besoin d'avoir une contre-image: elle pousse la génération antérieure à préciser sa propre image contre laquelle elle va elle-même se constituer.

D'un côté il y a donc les adolescents et de l'autre des adultes qui arrivent au milieu de la vie et pour lesquels j'ai proposé le terme de maturescence, par analogie à adolescence. Adolescence et maturescence sont deux phases critiques qui correspondent à cette confrontation des générations. La maturescence est une phase significative du parcours de vie qui émerge, a émergé, au xxe siècle, notamment sous l'effet des transformations sociales et économiques, et sous l'effet de l'allongement de l'espérance de vie: en atteignant le milieu de la vie, on a encore un nombre d'années significatif à vivre qui autorise des projets, des changements..., voire une crise (les difficultés sociales et familiales aidant). Le milieu de la vie est en outre marqué par une situation sans précédent historique; il se trouve entre deux générations de taille quasi équivalente. 
Dans la structure d'âge pyramidale, les générations qui se succédaient n'avaient pas la même taille. Les générations les plus âgées étaient de taille beaucoup plus restreinte que les générations plus jeunes, de taille beaucoup plus importante. Or la succession de générations de taille comparable qui prévaut à notre époque modifie complètement le rapport de relève de génération dans le travail comme dans les autres domaines de la vie sociale, famille y compris.

Il y a là, donc, une situation nouvelle pour une génération qui, à la phase du milieu de la vie, se trouve entre deux générations de taille comparable avec lesquelles elle redéfinit complètement ses positions, avec la génération des jeunes qui s'autonomise et celle des parents qui entre dans la vieillesse ; il s'instaure aussi avec cette dernière des relations nouvelles, de dépendance parfois, peut-être pas toujours matérielle mais généralement sociale et psychique. Avant de terminer je voudrais dire encore quelques mots sur la dernière phase de vie qui est le grand âge, un nouvel âge défini par une institutionnalisation croissante. Les personnes du grand âge qui, de plus en plus nombreuses, font l'objet maintenant d'une prise en charge dont la professionnalisation va croissante au sein de structures spécifiques. Ce grand âge est défini aussi par un rapport très particulier aux autres générations. La dernière génération en effet symbolise d'une certaine façon un rempart contre la mort, et ce rôle est aussi dévolu au grand âge.

N'ayant plus le temps de conclure, j'ajouterai simplement que même lorsqu'on définit les phases de vie par des éléments institutionnels, on peut retrouver dans les institutions mises en place un rôle de médiation dans les rapports entre générations. L'indemnité viagère de départ, n'est-elle pas aussi une façon de gérer les conflits de générations dans l'agriculture en incitant les plus âgés à se retirer? L'institutionnalisation du cours de vie revient alors à la gestion institutionnalisée des rapports de générations.

\section{NOTES}

1. S. N. Eisenstadt, From Generation to Generation. Age Groups and Social Structure, Glencoe, IL, Free Press, 1956, 357 p. (rééd. New Brunswick, NJ, Transaction, 2003). NdlR. 